\title{
A Range-assisted Detection Protocol against Replication Attacks in WSNs
}

\author{
Mingxi Li, Yan Xiong, Wenchao Huang and Xiancun Zhou, Xuangou Wu \\ School of Computer Science and Technology \\ University of Science and Technology of China, USTC \\ Hefei, China, 232007
}

keeperlmx@gmail.com,yxiong@ustc.edu.cn, huangwc@ustc.edu.cn, zhouxcun@mail.ustc.edu.cn,wxgou@ustc.edu.cn

\begin{abstract}
Wireless sensor networks (WSNs) consist of a large number of sensor nodes which are deployed without selfdefense capability. The node replication attack is a specific attack mode to WSN and the detection of node replication attacks in WSNs became a fundamental problem. In this paper, we proposed a range-assisted distributed protocol (RADP) to detect node replication attacks in WSNs. The contributions of this work are threefold. First, we showed that the known solutions do not completely meet our requirements. Second, we tried to use wireless ranging information to detect node replication attacks. Third, we proposed a novel protocol for the detection of node replication attacks and Extensive theoretical analysis and experiment verified that it can maintain a high detection probability with low accuracy ranging among nodes.
\end{abstract}

Keywords-wireless sensor networks; replication attacks; detection protocol; range-assisted.

\section{INTRODUCTION}

A Wireless Sensor Network (WSN) can be deployed in harsh environments to satisfy both military and civil applications, where staff is not easy to stay [1], [2]. With the development of wireless sensor networks, building a wireless sensor networks requires suitable security protocols [3] Replication attack (or clone attack) is a common attack mode in WSNs [4]-[5]. Replicas can launch a variety of attacks such as creating a black hole or initiating a wormhole attack [6]-[8]. There are a number of solutions for detecting node replication attacks in wireless sensor networks [9], [10].

By analyzing different characteristic data of networks, distributed solutions can be simply divided into three types:

(1) Using time-location claims is a simple resolution for detection of replication attacks in WSNs [11];

(2) The other resolution can protect WSN from replication attacks by data encryption [12];

(3) We can also protect a wireless sensor network by using other characteristic data of networks [13].

In this paper, a range-assisted detection protocol (RADP) is proposed for detecting node replication attacks in wireless sensor networks without other system information but the distances between nodes.

The rest of this paper is organized as follows: Some important technologies and thesis of WSNs has been introduced in Section II; Section III described the protocol in detail; Section IV is the analysis of the protocol; we show some experiment results in Section V; finally, conclusions and future work to our research are given in Section VI.

\section{RELATED WORK}

\section{A. Assumptions}

We assumed that sensor nodes in the network have unique identification which cannot be created (Newsome et al. describe several techniques to prevent the adversary from deploying node with arbitrary IDs [14]).

Since the main focus of this paper is to provide a solution to detect node replication attack, we assumed that a message fresh mechanism is available, which has been adopted in our protocols to prevent replication attacks in WSNs.

\section{B. Adversary model}

Adversaries have the capability of capturing and compromise a limited number of legitimate nodes in our problem settings. Thus, the replicas can easily participate in the network operation without being identified.

C. Notation

To clarity, Table 1 lists the symbols and notations.

TABLE I. SYMBOLS AND NOTATIONS

\begin{tabular}{|c|l|}
\hline$n$ & Number of nodes in network \\
\hline$S$ & The area of the region \\
\hline$x, x^{\prime}$ & A compromised node and its replica \\
\hline$x_{i}$ & The unique identity of node i \\
\hline$R$ & The transmission range of each node \\
\hline$R_{c}$ & $\begin{array}{l}\text { The maximum distance between node and its } \\
\text { close neighbors }\end{array}$ \\
\hline$R_{f}$ & $\begin{array}{l}\text { The minimum distance between node and its far } \\
\text { neighbors }\end{array}$ \\
\hline$\alpha$ & The maximum deviation of RSSI (by training) \\
\hline
\end{tabular}

\section{THE RADP}

\section{(Definition 1) Categorizing neighbours:}

We supposed that node $a$ is a neighbour of node $b$ (that is $\left|x_{a}-x_{b}\right| \leq R$ ). If $\left|x_{a}-x_{b}\right| \leq R_{c}$, they are a pair of close neighbours, and if $R_{f} \leq\left|x_{a}-x_{b}\right| \leq R$, they are called a pair of far neighbours. 
(Definition 2) Calculating $R_{c}$ and $R_{f}$ :

We express $\alpha$ as the maximum ranging deviation. To consider the impact of RSSI deviation, we can definite $R_{c}=R / 2-\alpha$ and $R_{f}=R / 2+\alpha$ then $\alpha<R / 2$.

(Definition 3) Constructing the detection information table of neighbours:

All of its neighbours' information is stored in the neighbour-information table (as show in Table 2).

TABLE II. NEIGHBOUR-INFORMATION TABLE

\begin{tabular}{|c|c|c|c|c|c|}
\hline Flag & 01 & 00 & 00 & $\ldots$ & 00 \\
\hline Node ID & 06 & 09 & 08 & $\ldots$ & 13 \\
\hline
\end{tabular}

By comparing nodes' neighbour-information tables, we can detect replication attacks.

\section{A. LUC: Local Unique Criterion}

As shown in Fig.1, when the replica $x^{\prime}$ and node $X$ can detect each other $\left(\left|x-x^{\prime}\right| \leq R\right)$, LUC come into force.

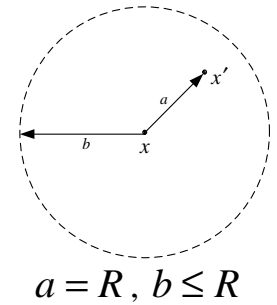

Figure 1. LUC: Local Unique Criterion

\section{B. NUC: Neighbour Unique Criterion}

We can find a node $x_{k}$ when both replica $x^{\prime}$ and compromised node $x$ be detected, meanwhile $x^{\prime}$ and $x$ are different kind neighbours of $x_{k}$ as shown in Fig. 2 .

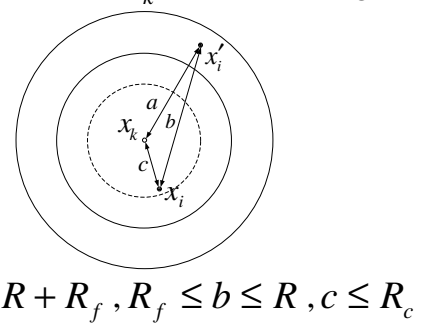

Figure 2. NUC: Neighbour Unique Criterion

Then NUC would be divided into two symmetrical situations described as the following:

(Situation1)

$$
R<\left|x-x^{\prime}\right|<R+R_{f}, R_{f} \leq\left|x-x_{k}\right| \leq R \text { and }
$$$$
\left|x^{\prime}-x_{k}\right| \leq R_{c} \text {; }
$$

(Situation2)

$$
\begin{aligned}
& \quad R<\left|x-x^{\prime}\right|<R+R_{f}, R_{f} \leq\left|x^{\prime}-x_{k}\right| \leq R \text { and } \\
& \left|x-x_{k}\right| \leq R_{c}
\end{aligned}
$$

\section{GUC: Global Unique Criterion}

As shown in Fig.3, when $x_{k_{i}}$ and $x_{k_{j}}$ find a same identification ( $x^{\prime}$ or $X$ ) as a close neighbour but they cannot detect each other $\left(\left|x_{k_{i}}-x_{k_{j}}\right|>R\right.$ ), GUC come into force..

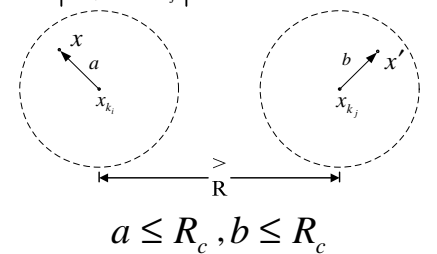

Figure 3. GUC: Global Unique Criterion

\section{ANALYSIS}

\section{A. $P_{1}$ : The Probability of LUC}

Because nodes are independent uniformly distributed, the distributed probability of $x_{i}$ can be describe as:

$$
f\left(x_{i}\right)= \begin{cases}\frac{1}{S}, & x_{i} \in S_{r e} \\ 0, & x_{i} \notin S_{r e}\end{cases}
$$

According to the definition of LUC $\left(\left|x-x^{\prime}\right| \leq R\right)$, the probability of LUC is

$$
P_{1}=\iint_{\left|x-x^{\prime}\right| \leq R} f(x) \cdot f\left(x^{\prime}\right) d S_{r e}
$$

Substituted (1) into (2), we can define $P_{1}$ :

$$
P_{1}=\iint_{\left|x-x^{\prime}\right| \leq R} \frac{1}{S} d S_{r e}=\frac{\pi R^{2}}{S}
$$

B. $P_{2}$ : The Detection Probability of NUC

\section{(Situation 1)}

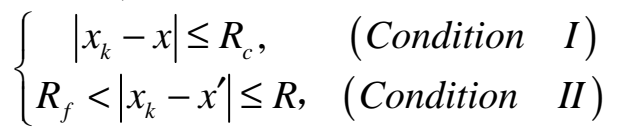

\section{(Situation 2)}

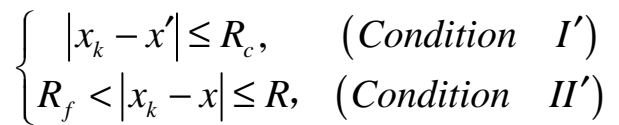

As given in (2), the probability of condition $I$ (or condition $I^{\prime}$ ) can be define as

$$
P(I)=\iint_{\left|x_{k}-x\right| \leq R_{c}} f\left(x_{k}\right) \cdot f(x) d S_{r e}=\frac{\pi(R-2 \alpha)^{2}}{4 s}
$$


The probability of condition $I I$ (or condition $I^{\prime}$ ) can be define as

$$
P(I I)=\iint_{R_{f}<x_{k}-x^{\prime} \leq R} f\left(x_{k}\right) \cdot f\left(x^{\prime}\right) d S_{S_{r e}}=\frac{\pi R^{2}-\pi\left(\frac{R}{2}+\alpha\right)^{2}}{s}
$$

The probability of situation 1 (or situation 2 ) is

$$
P(I) \cdot P(I I)=\frac{\pi^{2}(R-2 \alpha)^{3}(3 R+2 \alpha)}{16 s^{2}}
$$

Similarly, we can obtain $P\left(I^{\prime}\right) \cdot P\left(I I^{\prime}\right)$. Assumed $P_{2}^{\prime}$ is the probability of NUC, it can be defined as

$$
\begin{aligned}
P_{2}^{\prime} & =\left[P(I) P(I I)+P\left(I^{\prime}\right) P\left(I I^{\prime}\right)\right] \cdot\left(1-P_{1}\right) \\
& =\frac{\pi^{2}(R-2 \alpha)^{3}(3 R+2 \alpha)}{8 s^{3}} \cdot\left(s-\pi R^{2}\right)
\end{aligned}
$$

According to Bernoulli equation, we can define the detection probability of NUC as (8).

$$
P_{2}=1-\left(1-P_{2}^{\prime}\right)^{n-1}
$$

\section{C. $P_{3}$ : The Detection Probability of GUC}

\section{(Situation 1)}

$$
\left\{\begin{array}{l}
\left|x_{k_{1}}-x\right| \leq R_{c}, \quad(\text { Condition } I) \\
\left|x_{k_{2}}-x^{\prime}\right| \leq R_{c}, \quad(\text { Condition } I I) \\
\left|x_{k_{1}}-x_{k_{2}}\right|>R, \quad(\text { Condition }
\end{array}\right.
$$

(Situation 2)

$$
\left\{\begin{array}{l}
\left|x_{k_{1}}-x^{\prime}\right| \leq R_{c}, \quad\left(\text { Condition } I^{\prime}\right) \\
\left|x_{k_{2}}-x\right| \leq R_{c}, \quad\left(\text { Condition } I I^{\prime}\right) \\
\left|x_{k_{1}}-x_{k_{2}}\right|>R, \quad\left(\text { Condition } I I I^{\prime}\right)
\end{array}\right.
$$

Similar to the equation (4), the probabilities of condition $I$, condition $I I$ and condition $I I I$ can be defined as follows.

$$
\begin{aligned}
& P(I)=\iint_{\left|x_{k_{1}}-x\right| \leq R_{c}} f\left(x_{k_{1}}\right) \cdot f(x) d S_{r e}=\frac{\pi(R-2 \alpha)^{2}}{4 s}(9) \\
& P(I I)=\iint_{\left|x_{k_{2}}-x^{\prime}\right| \leq R_{c}} f\left(x_{k_{2}}\right) \cdot f\left(x^{\prime}\right) d S_{r e}=\frac{\pi(R-2 \alpha)^{2}}{4 s} \\
& P(I I I)=\iiint_{\left|x_{k_{1}}-x_{k_{2}}\right|>R} f\left(x_{k_{1}}\right) \cdot f\left(x_{k_{2}}\right) d S_{r e}=1-\frac{\pi R^{2}}{s}
\end{aligned}
$$

Considering that condition $I, I I$ and $I I I$ are independent of each other, so the probability of situation 1 (or situation 2) is

$$
P(I) \cdot P(I I) \cdot P(I I I)=\frac{\pi^{2}(R-2 \alpha)^{4}}{16 s^{3}}\left(s-\pi R^{2}\right)
$$

Similarly, we can obtain $P\left(I^{\prime}\right) \cdot P\left(I I^{\prime}\right) \cdot P\left(I I I^{\prime}\right) . P_{3}^{\prime}$ is the probability of NUC, it can be defined as

$$
\begin{aligned}
P_{3}^{\prime} & =\left[P(I) P(I) P(I I)+P\left(I^{\prime}\right) P\left(I^{\prime}\right) P\left(I^{\prime}\right)\right] \cdot\left(1-P_{1}\right) \cdot\left(1-P_{2}\right) \\
& =\frac{\pi^{2}(R-2 \alpha)^{4}}{8 s^{4}}\left(s-\pi R^{2}\right)^{2} \cdot\left(1-P_{2}\right)^{n-1}
\end{aligned}
$$

According to Bernoulli equation, $P_{3}$ can be describe as

$$
P_{3}=1-\left(1-P_{3}^{\prime}\right)^{C_{n-1}^{2}}
$$

In conclusion, we can obtain the total detection probability $P$ as the sum of $P_{1}, P_{2}$ and $P_{3}$.

$$
P=\frac{\pi R^{2}}{s}-\left(1-P_{2}^{\prime}\right)^{n-1}-\left(1-P_{3}^{\prime}\right)^{C_{n-1}^{2}}+2
$$

\section{EVALUATION}

\section{A. Simulation Evaluation}

To verify the feasibility of the RADP, we ran it in NS2 with $s=10000, n=200,1 \leq R \leq 10$ and $\alpha=\{0.1 \times$ Distance $, 0.2 \times$ Distance, $0.3 \times$ Distance $\}$ as shown in Fig.4.
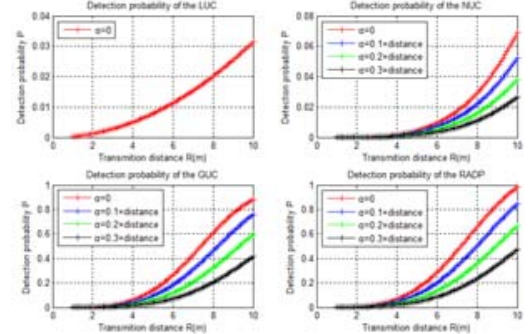

Figure 4. Detection probability of certain criterion with different $\alpha$

The simulation results verified that the RADP is more efficient than the RBDM [15] as shown in Fig.5.

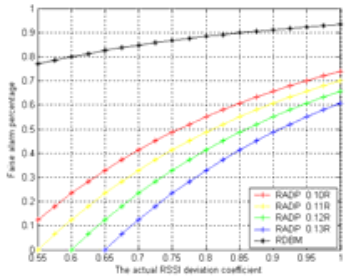

Figure 5. The percentage of false alarm in RADP and RBDM

\section{B. Real System Evaluation}

We run the RADP in a WSN experiment system called GAINS3 as shown in Fig.6.

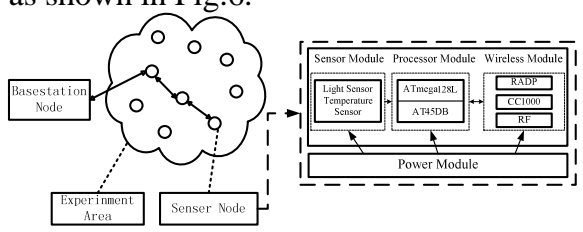

Figure 6. GAINS3 experiment system of WSN 
We set an experiment area $(s=10 \mathrm{~m} \times 8 \mathrm{~m})$ and deploy 30 nodes in it as shown in Fig.7.

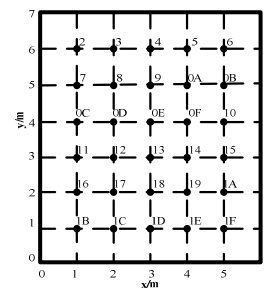

Figure 7. Real system experiment setting

The detection range of nodes is $5 \mathrm{~m}$ and the deviation is $\underline{0.5 \mathrm{~m}}$. All nodes run the RADP as Algorithm 1 .

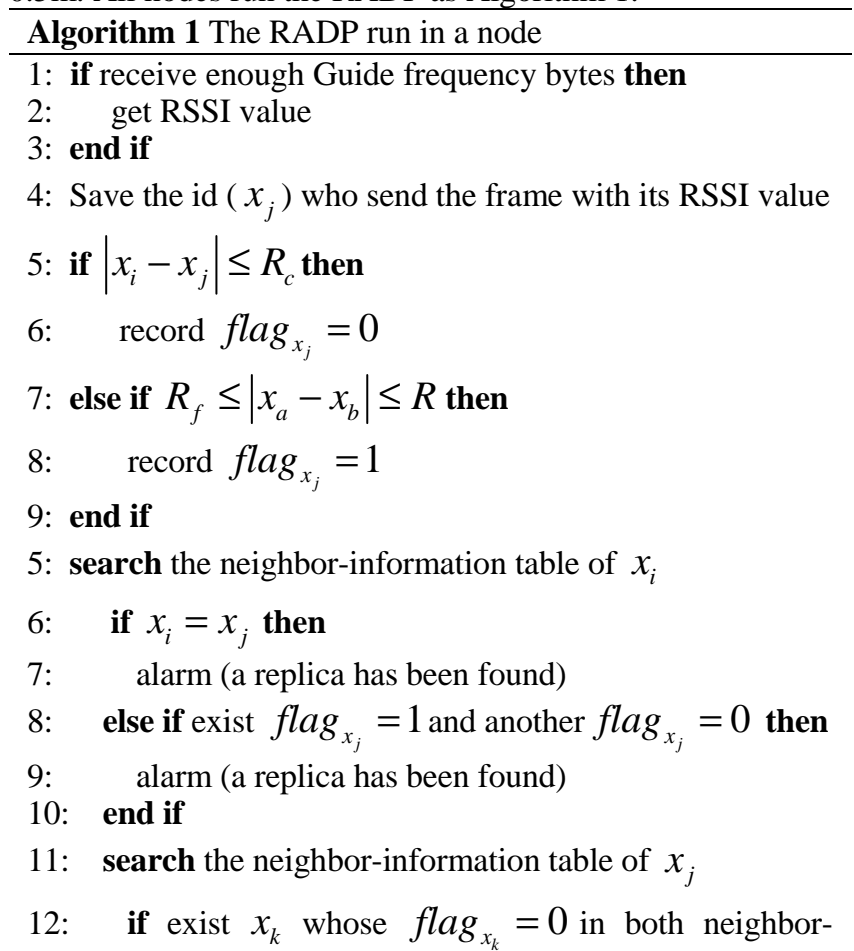

information tables of $x_{j}$ and $x_{i}$, but $x_{i}$ and $x_{j}$ is not a pair of neighbors then

13: $\quad$ alarm (a replica has been found)

14: end if

15: end search

\section{6: end search}

Our real system experiment result confirmed that the RADP is more suitable for the network comprising of a large number of nodes just like the WSN.

\section{CONCLUSION}

The RADP can be used as not only an independent protocol but also a sub-protocol of any other communication protocol. Our theoretical analysis and simulation results demonstrated that the RADP achieves excellent detection performance, low communication and false alarm percentage, without system synchronization time, precise node localization and other additional information.

\section{ACKNOWLEDGMENT}

This work was supported in part by the National Natural Science Foundation of China under grant No.61170233, No.61232018, No.61272472, No.61202404, No.61272317 and China Postdoctoral Science Foundation No.2011M501060.

\section{REFERENCES}

[1] I.F. Akyildiz, W. Su, Y. Sankarasubramaniam, and E. Cayirci. Wireless Sensor Networks: A Survey. Int'l J. Computer and Telecomm.Networking, vol. 38, no. 4, pp. 393-422, 2002.

[2] Xiangqian, C., Makki, K., Kang, Y., and Pissinou, N. SensorNetwork Security: a Survey, Communications Surveys Tutorials,IEEE , vol.11, no.2, pp.52-73, 2009.

[3] Haowen, C., and Perrig, A. 2003. Security and Privacy in SensorNetworks. Computer. vol.36, no.10, pp. 103-105.

[4] S. Zhu, S. Setia, and S. Jajodia. LEAP: efficient security mechanisms for large-scale distributed sensor networks. In CCS '03, pages 62-72, 2003.

[5] Conti, M., Di Pietro, R., Mancini, L. V., and Mei, A. A randomized, efficient, and distributed protocol for the detection of node replication attacks in wireless sensor networks. MobiHoc '07. ACM, New York, NY, pp. 80-89, 2007.

[6] S. Capkun and J.-P. Hubaux. Secure positioning of wireless devices with application to sensor networks. In INFOCOM '05, pages 19171928, 2005.

[7] Y. C. Hu, A. Perrig, and D. B. Johnson. Packet leashes: a defense against wormhole attacks in wireless networks. In INFOCOM '03, pages 1976-1986, 2003.

[8] Mauro Conti, Roberto Di Pietro, Luigi Vincenzo Mancini, and Alessandro Mei. Distributed Detection of Clone Attacks in Wireless Sensor Networks. IEEE Transactions on Dependable and secure computing, vol.8, no.5, pp. 685-698.September/October 2011.

[9] Mauro Conti, Roberto Di Pietro, Luigi V. Mancini, and Alessandro Mei. Requirements and Open Issues in Distributed Detection of Node Identity Replicas in WSN. In Proceedings of the 2006 IEEE International Conference on Systems, Man, and Cybernetics, pp.1468-1473. Taipei, Taiwan. October 8-11, 2006.

[10] B. Parno, A. Perrig, and V. Gligor. Distributed detection of node replication attacks in sensor networks. In Security and Privacy, 2005 IEEE Symposium on, pages 49-63. Ieee, 2005.

[11] Jadliwala M., Sheng Zhong, Upadhyaya S.J., Chunming Qiao,Hubaux J.-P. Secure Distance-Based Localization in the Presence of Cheating Beacon Nodes. IEEE Transactions on Mobile Computing, vol.9, no.6, pp.810-823, June 2010.

[12] Sarmad Ullah Khan, Luciano Lavagno, and Claudio Pastrone. A Key Management Scheme Supporting Node Mobility in Heterogeneous Sensor Networks. In ICET 2010, pp. 364-369, 2010.

[13] Heesook Choi, Sencun Zhu, Thomas F. La Porta. SET: Detecting node clones in Sensor Networks. In Proceedings of the 3rd International Conference on Security and Privacy in SecureComm 2007, pp. 341-350,2007.

[14] Newsome, J., Shi, E., Song, D., Perrig, A. , The Sybil attack in sensor networks: analysis defenses. The Information Processing in SensorNetworks (IPSN2004), pp. 259- 268, 26-27 April 2004.

[15] HUANG Jian, XIONG Yan, LI Mingxi, MIAO Fuyou. A Rangebased Detection Method of Replication Attacks in Wireless Sensor Networks. In ICICN 2012, vol. 27, pp. 120-127, 26-28 February 2012. 